\title{
Further data on the association between Helicobacter pylori infection and primary open-angle glaucoma
}

This article was published in the following Dove Press journal:

Clinical Ophthalmology

10 February 2012

Number of times this article has been viewed

\section{Christos Zavos \\ Jannis Kountouras}

Department of Medicine, Second Medical Clinic, Aristotle University of Thessaloniki, Ippokration Hospital, Thessaloniki, Greece
Correspondence: Jannis Kountouras

8 Fanariou St, Byzantio, 55I33,

Thessaloniki, Greece

$\mathrm{Tel}+302310892238$

Fax +30 2310992794

Email jannis@auth.gr
We herein wish to provide data additional to those reported in our previous review (Tsolaki et $\mathrm{al}^{1}$ ) concerning the association between Helicobacter pylori (H. pylori) infection and primary open-angle glaucoma. These data were not available in the time period when the review paper was submitted and accepted for publication, and we believe that they need to be added because they further reinforce this association.

In our new study published this year, ${ }^{2}$ we show for the first time the existence of $H$. pylori bacteria in the trabeculum and iris specimens of glaucoma patients, thereby further supporting the role for $H$. pylori infection in the pathophysiology of primary open-angle glaucoma. Specifically, the study included 51 consecutive patients who underwent trabeculectomy for primary open-angle glaucoma not responsive to topical antiglaucoma therapy. The presence of H. pylori was established by upper gastrointestinal endoscopy and histology, or by a urea breath test in eight patients who either were deemed not suitable endoscopy candidates or refused to undergo endoscopy.

All the patients underwent a surgical trabeculectomy procedure to their eyes, during which tissue samples from the trabeculum, conjunctiva, and iris were immediately obtained, placed in tubes containing 10\% formalin, and submitted for histological examination. These specimens were stained using the Cresyl fast violet method (for detection of $H$. pylori organisms). In five patients, for whom gastric $H$. pylori histology was positive, we managed to identify $H$. pylori bacteria in the trabeculum and iris specimens histologically with Cresyl fast violet stain for the first time. The reason why H. pylori was not found in the trabeculum and iris of all primary openangle glaucoma patients who tested positive for $H$. pylori status could be explained, apart from the absence of $H$. pylori in the eye, by the very small size of the sample of tissue obtained and submitted for histopathology during trabeculectomy, or possibly by standard local antisepsis prior to surgery. Despite the small number of cases, the findings of this study are important because $H$. pylori bacteria have been detected for the first time in the trabeculum and iris of patients with primary open-angle glaucoma, confirming that the bacterium is present locally, and is possibly directly implicated in glaucomatous damage.

In a second study just accepted for publication, ${ }^{3}$ we obtained biopsy specimens at upper gastrointestinal endoscopy from 43 patients with primary open-angle glaucoma, which were then evaluated for the presence of $H$. pylori, for expression of genes involved in cell proliferation and apoptosis (Ki-67, p53, Bcl-2), and for indices of cellular immune surveillance (T lymphocytes [TLs] and B lymphocytes [BLs]). Of the 
43 patients eligible for upper gastrointestinal endoscopy, 90.7\% tested positive for $H$. pylori infection. Ki-67 was positively expressed in $81.25 \%$ of patients with $H$. pylori infection and in one patient without $H$. pylori infection. p53 was positively expressed in $31.25 \%$ of patients with $H$. pylori infection but not in those without $H$. pylori infection. Bcl-2 was positively expressed in $68.75 \%$ of patients with $H$. pylori infection and in one patient without $H$. pylori infection. $\mathrm{Ki}-67, \mathrm{p} 53$, and Bcl-2 were overexpressed in 19\%, 25\%, and $37.5 \%$, respectively, of patients with $H$. pylori infection, but none was overexpressed in the patients without $H$. pylori infection. The TL marker was positively expressed in all patients with $H$. pylori infection and in the one patient without H. pylori infection. The BL marker was positively expressed only in one patient with $H$. pylori infection. Therefore, our forthcoming article provides further evidence that the H. pylori-induced oncogenes, Ki-67, p53 and Bcl-2, as well as TL markers are involved in cell proliferation and apoptotic pathways, thereby contributing to glaucomatous neuropathy, with oncogenic potential. In this respect, and apart from the apoptotic processes involved in the pathophysiology of glaucomatous neuropathy, recent experimental data indicate that cell proliferation rather than astrocyte hypertrophy characterizes early pressure-induced optic nerve head injury, and the optic nerve head is the principal site of initial axonal injury in glaucoma. Furthermore, at the eye level, Ki-67 has been used as an index of melanoma proliferation and also as an index showing response to treatment with agents that inhibit the proliferation of Tenon's fibroblasts, decreasing excessive scarring after trabeculectomy. ${ }^{2}$

Specifically, we have also very recently provided an overview of the various pathophysiological mechanisms underlying the association between $H$. pylori infection and primary open-angle glaucoma ${ }^{4}$ which include: promoting platelet and platelet-leukocyte aggregation, also involved in the pathophysiology of glaucoma; releasing proinflammatory and vasoactive substances, including cytokines (interleukins-1, $-6,-8,-10$, and -12 , tumor necrosis factor alpha [TNF- $\alpha$ ], interferon-gamma), eicosanoids (leukotrienes, prostaglandins) and acute-phase proteins (fibrinogen, C-reactive protein) involved in vascular disorders and glaucoma; stimulating mononuclear cells to induce tissue factor-like procoagulant activity that converts fibrinogen into fibrin; causing the development of cross-mimicry between endothelial and $H$. pylori antigens; producing oxidative stress and circulating lipid peroxides; and in particular influencing the apoptotic process, parameters of which may also exert their own effects in the induction and/or progression of glaucoma and other neurodegenerative disorders (Guillain-Barré syndrome, Alzheimer's disease, Parkinson's disease) associated with both $H$. pylori infection and glaucoma.

Importantly, $H$. pylori infection and glaucoma share the Fas/FasL and mitochondria-mediated apoptotic pathways, thereby suggesting an apoptotic link in the pathophysiology of both diseases. In particular, increased endothelin-1 (a potent constrictor of arterioles and venules), nitric oxide, and inducible nitric oxide synthase levels are associated with $H$. pylori infection. Endothelin-1-induced anterior optic nerve vessel vasoconstriction and vascular tone modulation by nitric oxide in the ophthalmic artery may produce glaucomatous damage. Moreover, nitric oxide, a rapidly diffusing gas, is a potent neurotoxin that may facilitate apoptotic retinal ganglion cell death in glaucomatous optic neuropathy. Support for the consideration of nitric oxide neurotoxicity in glaucoma is provided by experimental evidence demonstrating that retinal ganglion cell apoptosis is attenuated by neutralizing antibodies against TNF- $\alpha$ or by selective inhibitors of inducible nitric oxide synthase, thereby suggesting that the inhibitors of TNF- $\alpha$ or of the inducible isoform (NOS-2) may provide novel therapeutic targets for neuroprotection in the treatment of glaucomatous optic neuropathy.

In addition, systemic $H$. pylori-induced oxidative damage may be the mechanism which links oxidative stress, $H$. pylori infection, and the apoptotic damage to the trabecular meshwork and optical nerve head that results in glaucoma. In this regard, oxidative stress is an essential underlying cause of neuroinflammatory and neurodegenerative diseases, including glaucoma and the blood-brain barrier damage connected to these diseases; oxidative stress activates protein tyrosine kinase and matrix metalloproteinases, resulting in bloodbrain barrier dysfunction. ${ }^{4}$

H. pylori infection, by releasing several inflammatory mediators, ${ }^{5}$ could induce breakdown of the blood-brain/ blood-ocular barriers, thereby being involved in the pathogenesis of neuropathies, including glaucoma. ${ }^{5}$ For instance, H. pylori could indirectly affect the brain through the release of TNF- $\alpha$ acting at a distance; TNF- $\alpha$ is involved in blood-brain barrier disruption through upregulation of matrix metalloproteinases. Furthermore, circulating antibodies for $H$. pylori might also enter the aqueous circulation due to disruption of the blood-brain/blood-ocular barriers, possibly contributing to the pathophysiology of glaucoma; when serum-specific antibodies access the brain, they are capable of killing retinal cells. ${ }^{5}$ Likewise, an influx of $H$. pylori-infected monocytes, owing to defective autophagy 
resulting in $H$. pylori replication in autophagic vesicles, through the disrupted blood-brain/blood-ocular barrier, might lead to glaucoma neuropathy. H. pylori VacA cytotoxin promotes intracellular survival of the bacterium and modulates host immune responses. ${ }^{5}$ In addition, because the oral cavity might act as a permanent reservoir for $H$. pylori, this bacterium may reach the eye through the nasal cavity, causing ophthalmic pathologies, possibly including glaucoma. ${ }^{5}$

Finally, it is important to note that studies conducted in Korea, China, India, Turkey, and Iran have also reported an association between $H$. pylori infection and glaucoma. ${ }^{2}$

\section{Disclosure}

The authors report no conflicts of interest in this work.

\section{References}

1. Tsolaki F, Gogaki E, Sakkias F, et al. Helicobacter pylori infection and primary open-angle glaucoma: is there a connection? Clin Ophthalmol. 2012;6:45-47.

2. Zavos C, Kountouras J, Sakkias G, Venizelos I, Deretzi G, Arapoglou S. Histological presence of Helicobacter pylori bacteria in the trabeculum and iris of patients with primary open-angle glaucoma. Ophthalmic Res. 2012;47(3):150-156.

3. Zavos C, Kountouras J, Sakkias G, et al. Oncogenes' expression in Greek patients with primary open-angle glaucoma in association with Helicobacter pylori status. Immunogastroenterology. 2012:1(1). In press. DOI: 10.7178/ig.1.1.1.

4. Kountouras J, Zavos C, Deretzi G, et al. Neuroprotection in glaucoma: is there a future role of Helicobacter pylori eradication? Exp Eye Res. 2011;92(5):436-438.

5. Kountouras J, Zavos C, Sakkias G, et al. Helicobacter pylori infection as a risk factor for both primary open-angle glaucoma and pseudoexfoliative glaucoma in Thessaloniki Eye Study. Am J Ophthalmol. 2011; 152(6):1079-1080.
Clinical Ophthalmology

\section{Publish your work in this journal}

Clinical Ophthalmology is an international, peer-reviewed journal covering all subspecialties within ophthalmology. Key topics include: Optometry; Visual science; Pharmacology and drug therapy in eye diseases; Basic Sciences; Primary and Secondary eye care; Patien Safety and Quality of Care Improvements. This journal is indexed on

Submit your manuscript here: http://www.dovepress.com/clinical-ophthalmology-journal

\section{Dovepress}

PubMed Central and CAS, and is the official journal of The Society of Clinical Ophthalmology (SCO). The manuscript management system is completely online and includes a very quick and fair peer-review system, which is all easy to use. Visit http://www.dovepress.com/ testimonials.php to read real quotes from published authors. 